\title{
LOW-VOLTAGE SILICON PHOTONIC MEMS SWITCH WITH VERTICAL
} ACTUATION

\author{
Hamed Sattari ${ }^{1}$, Alain Y. Takabayashi ${ }^{1}$, Pierre Edinger ${ }^{2}$, Peter Verheyen ${ }^{3}$, Kristinn B. Gylfason ${ }^{2}$, \\ Wim Bogaerts ${ }^{3,4}$, and Niels Quack ${ }^{1}$ \\ ${ }^{1}$ École Polytechnique Fédérale de Lausanne, 1015 Lausanne, Switzerland \\ ${ }^{2} \mathrm{KTH}$ Royal Institute of Technology, School of Electrical Engineering and Computer Science, Micro \\ and Nanosystems, Stockholm, Sweden \\ ${ }^{3}$ Interuniversity Microelectronics Centre (IMEC), Leuven, Belgium and \\ ${ }^{4}$ Ghent University-imec, Photonics Research Group, Department of Information Technology, Ghent, \\ Belgium
}

\begin{abstract}
We present a vertically movable silicon photonic MEMS switch realized in IMEC's standard silicon photonics platform followed by a dedicated postprocessing for MEMS release. The device has six optical ports, which enable four switching configurations with a safe electrical isolation of the switch's actuator. A low actuation voltage of $3.75 \mathrm{~V}$ is required to efficiently switch the optical signal from the drop port to the through port of the device. The device exhibits port extinctions of $16 \mathrm{~dB}$ and $26 \mathrm{~dB}$ at its $\mathrm{OFF}$ and $\mathrm{ON}$ states, respectively. With an insertion loss of $<2 \mathrm{~dB}$ and a broad $3 \mathrm{~dB}$-bandwidth $>35 \mathrm{~nm}$, this component paves the way for low-power scalable circuits in MEMS-enabled silicon photonics.
\end{abstract}

\section{KEYWORDS}

Silicon Photonic MEMS; Optical Switch; Directional Coupler.

\section{INTRODUCTION}

In the past decade, the manufacturing of large-scale photonic integrated circuits (PIC) has been made possible at the foundry level. Silicon photonics platforms have excelled over other platforms in terms of standardization thanks to the compatibility with well-developed CMOS manufacturing processes and availability of high throughput tools. Several standard building blocks including passives, such as arrayed waveguide gratings or interferometers, and actives, such as high-speed photodetectors or modulators, are available in silicon photonics process design kits (PDK) [1]. Among the essential components for large-scale photonic integrated circuits, optical switches and phase shifters are the workhorses for a multitude of functions. At the same time, the target markets for platform-based PICs, e.g. telecommunications and datacenters, demand large-scale integration and power-efficient circuits to address their emerging needs [2], [3]. Currently, silicon photonic switches are typically based on either thermo-optic or plasma dispersion effects. Thermo-optical switches are power-hungry and occupy a relatively large area. They also suffer from thermal cross talk, which further limits their use in large-scale circuits. Switches based on the plasmadispersion effect are fast (ns switching time), and quite compact ring resonator-based designs have been proposed. However, due to the intrinsic photon-carrier interaction, the plasma dispersion effect typically induces substantial optical losses, and therefore present limitations for large- scale integration [2], [4].

Novel solutions such as plasmonic-based modulators have been demonstrated recently, yet further improvements are required to decrease the insertion loss and increase extinction ratio per device before being implemented in densely integrated circuit arrays [4]. In contrast, photonic MEMS have already shown their potential for addressing the challenges of large integrated photonic circuits, in particular with regard to scalability and power efficiency [5], [6]. In a photonic MEMS device, the optical signal is altered by the physical displacement of the waveguides, which provides a more efficient modulation scheme when compared to the thermo-optic and plasma dispersion effects. This approach enables more compact switches and with electrostatic actuation, there is no additional optical loss due to free carrier interaction [6]. Furthermore, photonic MEMS can be designed to be latchable, thereby enabling non-volatile states providing unique opportunities for programmable photonic circuits [7], [8].

Hitherto, various silicon photonic MEMS components have been realized either on simple silicon on insulator (SOI) substrates or on customized device layer stacks [5], [6]. The main concept of silicon photonic MEMS switching relies on the electrostatic modulation of a gap between coupled waveguides in a (directional) coupler or ring resonator system. As a successful demonstration of silicon photonic MEMS switch scalability, a $240 \times 240$ switch matrix has been demonstrated recently, using a fully customized multi-layer waveguide process [9]. However, to enjoy the full capability of an advanced silicon photonics technology platform with high performance building blocks (e.g. modulators and detectors), the photonic MEMS need to be integrated in the existing platform stack.

Recently, we have demonstrated successful integration of a broadband suspended directional coupler [10], a phase shifter [11], and an optical single-pole-double-throw switch [12] in IMEC's well-established ISIPP50G platform [13]. So far, we have successfully implemented vertically movable and horizontally movable MEMS components in the platform. Our goal is to extend the library of standard components with additional high-performance photonic MEMS blocks [14], [15]. Doing so will enable new functionalities by integrating the MEMS elements next to other standard components in the same circuit. Here we report on the design, fabrication, and characterization of a vertically movable photonic MEMS switch in IMEC's ISIPP50G technology, followed by a simple postprocessing for releasing the MEMS devices. 


\section{MEMS SWITCH WORKING PRINCIPLE}

A three-dimensional representation of the silicon photonic MEMS switch is shown in Fig. 1. The switch encompasses three suspended directional couplers, two of which are stiff, and one which is soft, for a total of six optical ports.

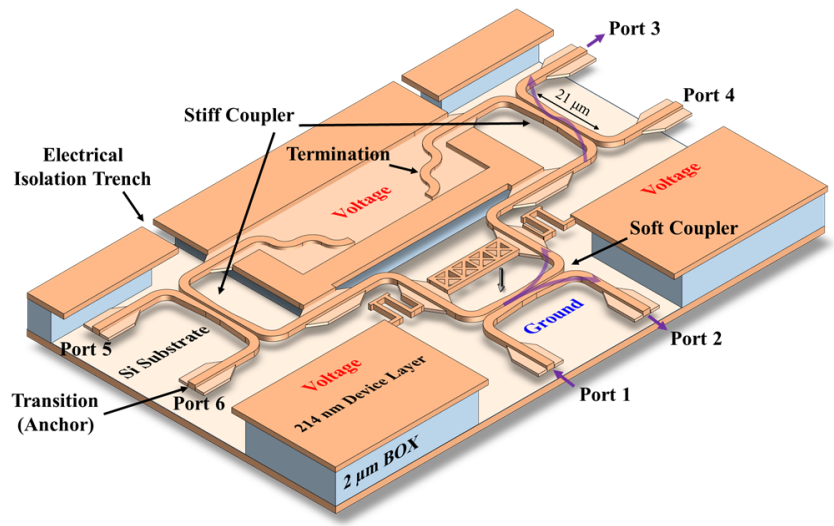

Figure 1: Schematic representation of the vertically movable silicon photonic MEMS switch. Dimensions are not to scale.

The soft directional coupler is anchored with a set of compliant springs while the stiff couplers are anchored by stiff shallow etched waveguide anchors. The device layer has a full thickness of $214 \mathrm{~nm}$ and a shallow etched slab thickness of $150 \mathrm{~nm}$ in the waveguide transitions and anchors. The switching is accomplished by applying a bias voltage between the silicon substrate and the device layer, which are separated by a $2 \mu \mathrm{m}$ air gap, that is defined by the buried oxide (BOX) layer thickness. In the active state of the switch, for relatively low voltages, the soft coupler moves towards the substrate while the stiff couplers remain in their original position. This action increases the air gap between the coupled waveguides in the soft coupler and leads to an efficient transfer of the optical signal to the through port.

A photonic MEMS switch can also be achieved with one single movable directional coupler. However, when integrating such a switch in a larger circuit, the electrically active parts of the device should be isolated from the neighboring devices, such as thermal phase shifters and photodetectors, so as to prevent electrical crosstalk and short circuits. Therefore, in our design, we fully decouple the active portion of the switch from the platform's device layer by implementing two additional directional couplers (stiff couplers) in the circuit. Although, this arrangement increases the device footprint slightly and might rise the total insertion loss, it provides the additional benefit of enabling extra switching configurations, which improves integration flexibility in larger circuits and waveguide meshes. Possible switching configurations are listed in Table. 1.

Table 1: Switching configurations for the silicon photonic MEMS switch with 6 optical ports.

\begin{tabular}{l|c|c|c} 
& Input & Through & Drop \\
\hline Configuration 1 & Port 1 & Port 2 & Port 3 \\
\hline Configuration 2 & Port 2 & Port 1 & Port 5 \\
\hline Configuration 3 & Port 3 & Port 4 & Port 1 \\
\hline Configuration 4 & Port 5 & Port 6 & Port 2
\end{tabular}

All three directional couplers have identical geometry. With a total length of $21 \mu \mathrm{m}$, they are composed of a pair of double-tapered waveguides separated by a 150 $\mathrm{nm}$ air gap. For the details of the photonic design, simulation, and characterization of the suspended directional coupler without MEMS actuators we refer to our recent publications [10], [15]. The six optical ports are terminated with waveguide transitions between fully etched and partially etched waveguides which then pass through the wall of the MEMS cavity to the oxide-clad waveguide components. The MEMS cavity itself is a deep opening within the back-end-of-line (BEOL) dielectric stack that provides local access to the silicon device layer. Removing the underlying BOX in the cavity then creates suspended devices. The transitions through the wall are optimized for low loss and reflection between the air-clad and oxide-clad regions [15]. The photonic MEMS switch unit cell has a footprint of $96 \mu \mathrm{m} \times 146 \mu \mathrm{m}$, and is designed to route optical signals within the telecommunication $\mathrm{C}$ band with TE polarization.

\section{FABRICATION PROCESS}

The manufacturing of the silicon photonic MEMS components is realized in two steps.

First, a full ISIPP50G standard platform fabrication process run is performed on wafer-level as offered by IMEC [13]. The MEMS devices are hereby structured as part of the standard process in the silicon photonics device layer, and integrated alongside standard library components such as heaters, electro-optical modulators, photodetectors, the standard metal routing and bond pad interfaces, etc. As part of the standard process, an opening in the back end of line stack is performed to expose the waveguides (EXPO module). This opening is performed over the silicon photonic MEMS devices.

Second, a custom MEMS release process is performed on die-level. The post-processing steps are executed at the EPFL center of MicroNanotechnology (CMi) as schematically shown in Fig. 2.
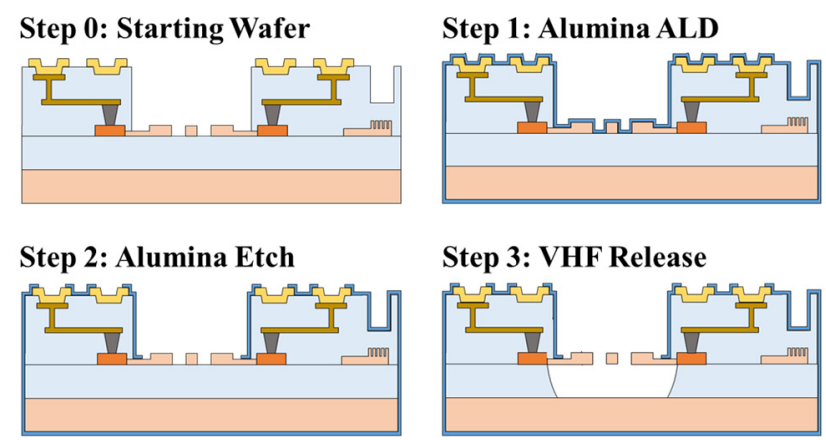

$\mathrm{Si} \square \mathrm{SiO}_{2} \square$ Doped Si

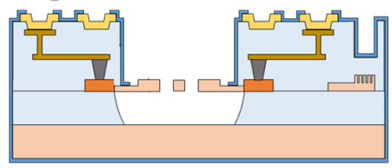

Figure 2: The post-processing steps for releasing the photonic MEMS.

For the post-processing, first, we deposit $20 \mathrm{~nm}$ of conformal alumina over the sample using an atomic layer deposition (ALD) technique (BENQ TSF200) which passivates the BEOL stack and follows the topography of its side walls. Next, we selectively pattern the thin passivation layer on the photonic MEMS components and the metal contact pads using a direct laser writing 
photolithography and a subsequent dry etching step. Opening alumina above the MEMS cavities defines the access windows to the MEMS components just before the last step. Finally, for releasing the MEMS, a vapour hydrofluoric acid (VHF) etching is performed to fully remove the $2 \mu \mathrm{m}$ thick BOX under the MEMS and realize suspended and movable structures.

An optical microscope image of the device in Fig.3.a reveals a uniform undercut front in the BOX, and the SEM image at Fig.3.b depicts a clean release step and the excellent quality of the suspended components.
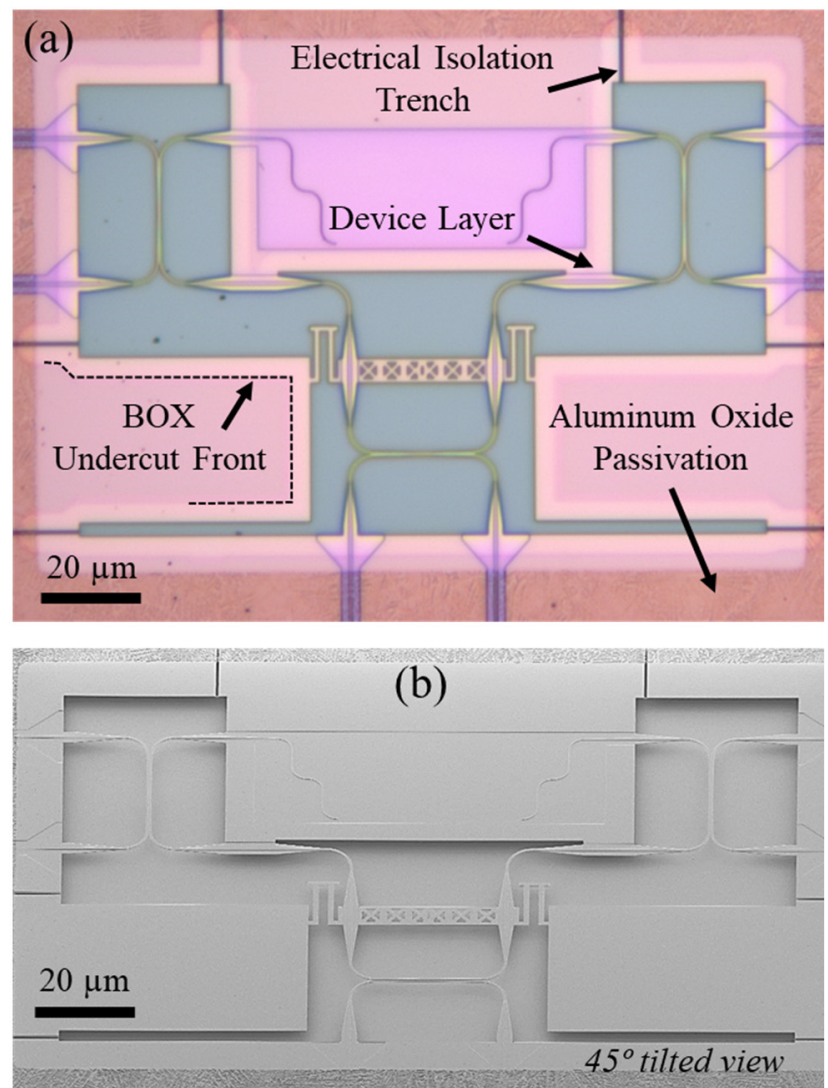

Figure 3: (a) Optical image of the photonic MEMS switch cavity. (b) SEM recording of the released switch. The device is cleanly released, and the suspended couplers waveguides are perfectly aligned.

\section{RESULTS AND DISCUSSION}

An optical microscope image of the released chip area with the photonic MEMS switch, contact pads, grating couplers, waveguides, and the metallization layers and a schematic representation of the characterization setup are shown in Fig.4.

The characterization setup consists of a tunable laser, a detector module, a single mode fiber array, and electrical probe tips. Light is coupled into and out of the chip using the finely position-tuned single mode fiber array. The sample is mounted on a stage with three degrees of freedom while the fiber array is controlled on a precision alignment stage with six degrees of freedom. This setup allows for a precise alignment of the fiber array to the integrated vertical grating coupler arrays on the photonic chip. A set of electrical probes are used to bias the MEMS components and ground the silicon substrate properly.
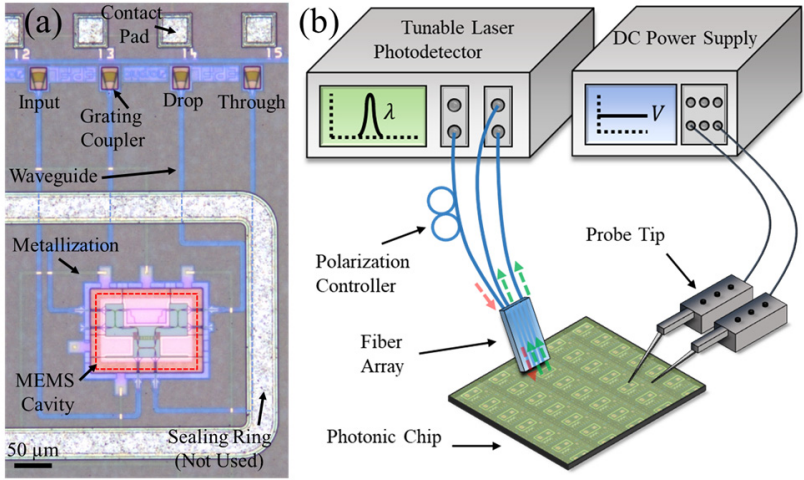

Figure 4: (a) Optical image of the released chip area. (b) Sketch of the integrated photonic MEMS component characterization setup.

The measurement results for the silicon photonic MEMS switch in configuration 1 (see Table 1 and Fig. 1) is shown in Fig. 5. The figure presents the drop and through ports' transmission spectra for the OFF and ON states of the switch. In the OFF state, which is the as-released state of the switch, the suspended couplers are in the cross state; therefore, injected light from the input port (port 1) efficiently couples to the drop port (port 3 ) after passing the soft coupler and one of the stiff couplers. Within this state, a port isolation of $16 \mathrm{~dB}$ is measured and the insertion loss is $\sim 1 \mathrm{~dB}$. By applying a low bias voltage of $3.75 \mathrm{~V}$, the soft directional coupler arm moves towards the silicon substrate and sufficiently decouples the directional coupler arms. This action leads to light transfer to the through port. The applied bias is enough to increase the vertical separation of the coupler arms by $>500 \mathrm{~nm}$, while at the same time maintaining a safe distance from the substrate to prevent collapse to the substrate, due to the pull-in effect. The stiff directional couplers as well as the inactive part of the device layer are grounded upon the actuation. The central operating wavelength of the device $\lambda=1550 \mathrm{~nm}$ perfectly coincides with our simulation results [10]. In the ON state, the device exhibits a high port isolation of $\sim 26 \mathrm{~dB}$ and an insertion loss of $<2 \mathrm{~dB}$. Thanks to the unique design of the suspended directional couplers, the device exhibits a $3 \mathrm{~dB}$ bandwidth $>35 \mathrm{~nm}$ within the telecommunication $\mathrm{C}$-band.

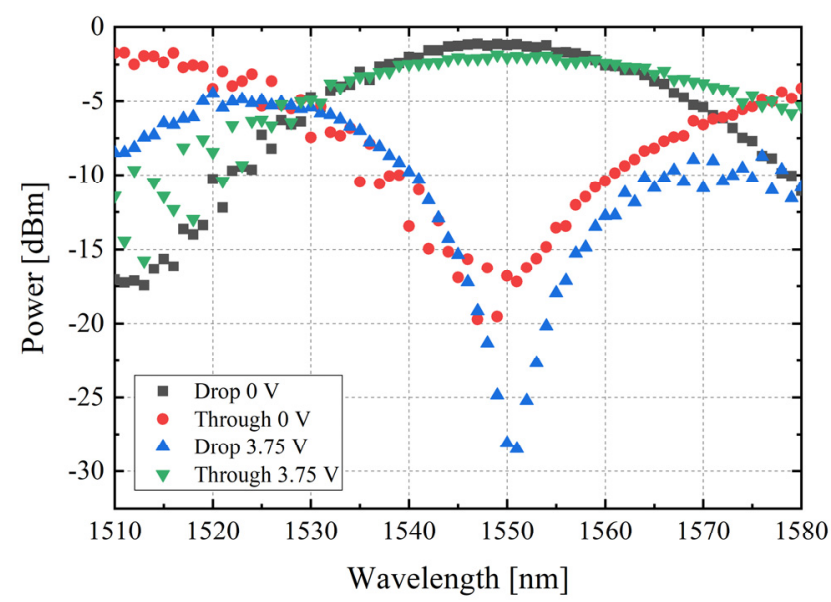

Figure 5: The drop and through ports' transmission spectra for the OFF $(0 \mathrm{~V})$ and $O N(3.75 \mathrm{~V})$ states of the switch. 
The device demonstrates larger port isolation in the ON state in comparison with the OFF state, as there is no coupled directional coupler in the light path for the $\mathrm{ON}$ state. In the OFF state, there are two directional couplers with the cross state in the light path; consequently, a portion of the light leaks to the through state, which we attribute due to fabrication imperfections affecting the initial air gap between the couplers. Identifying the origin of the insertion loss difference between the two states $(\mathrm{ON}$ and OFF) is not straightforward. In particular, the insertion loss includes a portion of the losses from the optical transitions and a portion from the directional couplers. We expect a lower insertion loss in the $\mathrm{ON}$ state when there is no coupled directional coupler in the light path. Nevertheless, due to possible fabrication imperfections, the optical signal undergoes a higher scattering loss when passing through the tapered single coupler arm in the OFF state. The insertion loss difference could also be attributed to a variation in performance of the individual passive elements such as the transitions, waveguides, and the grating couplers in the light paths for the ON and OFF states.

Note that for any other switching configuration presented in Table. 1, the optical signal passes two directional couplers in the OFF state and one coupler arm in the $\mathrm{ON}$ state, and insertion losses $<2 \mathrm{~dB}$ are expected for all the configurations.

\section{CONCLUSION}

We propose a design and demonstrate the functionality of a silicon photonic MEMS switch fabricated in the full device stack of IMEC's ISIPP50G platform with custom MEMS release post processing. The switch enables drop and through port isolations of $16 \mathrm{~dB}$ and $26 \mathrm{~dB}$ at OFF and ON states, respectively. The device is actuated with a low voltage of $3.75 \mathrm{~V}$ that displaces its vertically movable directional coupler. This device with a $3 \mathrm{~dB}$ bandwidth of $>35 \mathrm{~nm}$ and a relatively compact footprint of $96 \mu \mathrm{m} \times 146$ $\mu \mathrm{m}$ holds promises for a new generation of MEMS-enabled standard library components for silicon photonic platforms.

\section{ACKNOWLEDGEMENTS}

This project has received funding from the European Union's Horizon 2020 research and innovation program under grant No. 780283 (MORPHIC www.h2020morphic.eu). H. Sattari acknowledges funding from the Hasler Foundation under grant No. 17008, and Niels Quack from the Swiss National Science Foundation under grant No. 183717. The authors acknowledge the technical support of the CMi management and staff.

\section{REFERENCES}

[1] A. Rahim, T. Spuesens, R. Baets, and W. Bogaerts, "Open-Access Silicon Photonics: Current Status and Emerging Initiatives," Proceedings of the IEEE, vol. 106, no. 12, pp. 2313-2330, Dec. 2018.

[2] L. Thylén and L. Wosinski, "Integrated photonics in the 21 st century," Photonics Research, vol. 2, no. 2, p. 75, Apr. 2014.

[3] Q. Cheng, S. Rumley, M. Bahadori, and K. Bergman, "Photonic switching in high performance datacenters [Invited]," Optics Express, vol. 26, no. 12, p. 16022, Jun. 2018.
[4] C. Haffner et al., "Low-loss plasmon-assisted electrooptic modulator," Nature, vol. 556, no. 7702, pp. 483486, Apr. 2018.

[5] X. Tu, C. Song, T. Huang, Z. Chen, and H. Fu, "State of the Art and Perspectives on Silicon Photonic Switches," Micromachines, vol. 10, no. 1, p. 51, Jan. 2019.

[6] C. Errando-Herranz, A. Y. Takabayashi, P. Edinger, H. Sattari, K. B. Gylfason, and N. Quack, "MEMS for Photonic Integrated Circuits," IEEE Journal of Selected Topics in Quantum Electronics, vol. 26, no. 2, pp. 1-16, Mar. 2020.

[7] W. Bogaerts et al., "Building Large-Scale Programmable Photonic Circuits Using Silicon Photonic MEMS," in OSA Advanced Photonics Congress (AP) 2020 (IPR, NP, NOMA, Networks, PVLED, PSC, SPPCom, SOF) (2020), paper PsTh1F.1, Jul. 2020, p. PsTh1F.1.

[8] W. Bogaerts et al., "Programmable photonic circuits," Nature, vol. 586, no. 7828, Art. no. 7828, Oct. 2020.

[9] T. J. Seok, K. Kwon, J. Henriksson, J. Luo, and M. C. $\mathrm{Wu}$, "Wafer-scale silicon photonic switches beyond die size limit," Optica, vol. 6, no. 4, p. 490, Apr. 2019.

[10] H. Sattari et al., "Compact broadband suspended silicon photonic directional coupler," Opt. Lett., OL, vol. 45, no. 11, pp. 2997-3000, Jun. 2020.

[11] P. Edinger et al., "Compact low loss MEMS phase shifters for scalable field-programmable silicon photonics," in Conference on Lasers and ElectroOptics (2020), paper SM3J.2, May 2020, p. SM3J.2.

[12] A. Takabayashi et al., "Broadband Compact SinglePole Double-Throw Silicon Photonic MEMS Switch," in European Conference on Integrated Optics (ECIO) (2020), pp 1-3.

[13] M. Pantouvaki et al., "Active Components for $50 \mathrm{~Gb} / \mathrm{s}$ NRZ-OOK Optical Interconnects in a Silicon Photonics Platform," Journal of Lightwave Technology, vol. 35, no. 4, pp. 631-638, Feb. 2017.

[14] W. Bogaerts et al., "MORPHIC: programmable photonic circuits enabled by silicon photonic MEMS," in Silicon Photonics XV, Feb. 2020, vol. 11285, p. 1128503.

[15] N. Quack et al., "MEMS-Enabled Silicon Photonic Integrated Devices and Circuits," IEEE Journal of Quantum Electronics, vol. 56, no. 1, pp. 1-10, Feb. 2020.

\section{CONTACT}

*H.Sattari, tel: +41-21-6937737; hamed.sattari@epfl.ch 